\title{
Short communication: N-Acetylcysteine-mediated augmentation of $\beta$-lactam antibacterial activity against methicillin-resistant Staphylococcus aureus isolated from bovine mastitis cases
}

\section{Feng Yang, ${ }^{*}$ Shidong Zhang, Xiaofei Shang, Xurong Wang, Ling Wang, and Yan Sun*}

Engineering and Technology Research Center of Traditional Chinese Veterinary Medicine of Gansu Province, Key Lab of New Animal Drug Project of Gansu Province, Key Lab of Veterinary Pharmaceutical Development of Ministry of Agriculture, Lanzhou Institute of Husbandry and Pharmaceutical Sciences of Chinese Academy of Agricultural Science, Lanzhou 730050, P. R. China

\section{ABSTRACT}

The present study investigated the effects of $\mathrm{N}$-acetylcysteine (NAC) on $\beta$-lactam antibacterial activity against 20 methicillin-resistant Staphylococcus aureus (MRSA) isolates from bovine mastitis. Minimum inhibitory concentrations (MIC) were determined by the E-test method. The presence of $10 \mathrm{mM}$ NAC reduced the MIC of penicillin, ampicillin, oxacillin, cefoxitin, ceftazidime, and cefotaxime to MRSA. Importantly, the MIC of cefoxitin in MRSA in the presence of NAC was lower than the susceptible breakpoint of cefoxitin. The results provide a new way to use current $\beta$-lactam antibiotics combined with NAC against MRSA.

Key words: bovine mastitis, methicillin-resistant Staphylococcus aureus, $\mathrm{N}$-acetylcysteine, resistance

\section{Short Communication}

Methicillin-resistant Staphylococcus aureus (MRSA) is a major drug-resistant pathogen responsible for nosocomial infections in humans and IMI in cattle (Graveland et al., 2011). Of note, MRSA is resistant to most $\beta$-lactam antibiotics and multiple other antimicrobial classes currently available for mastitis therapy, which has major implications for current and future treatment options for this pathogen (Vanderhaeghen et al., 2010; Lee et al., 2018). $N$-Acetylcysteine (NAC) is an important modulator of antibiotic activity (Goswami and Jawali, 2010). Our previous study showed that NAC increased the antibacterial activity of penicillin and ampicillin against bovine mastitis Staphylococcus aureus (Yang et al., 2016), which were later con-

Received January 29, 2019.

Accepted April 15, 2019.

*Corresponding authors: yangfeng@caas.cn and 516845664@qq.com firmed as methicillin-susceptible Staphylococcus aureus (MSSA; data not shown). Here, we report the effect of NAC on $\beta$-lactam antibacterial activity against MRSA recovered from bovine mastitis cases.

The 20 MRSA isolates used in this study were isolated from cases of bovine mastitis in China. The MRSA were screened by using ChromID MRSA agar (BioMérieux, Marcy-l'Étoile, France) and confirmed by cefoxitin $(30 \mu \mathrm{g})$ disk diffusion method according to Clinical and Laboratory Standards Institute (CLSI) recommendations (CLSI, 2013). The effect of NAC (Sigma-Aldrich, Lyon, France) on the susceptibility of MRSA to $\beta$-lactam antibiotics (penicillin, ampicillin, oxacillin, cefoxitin, ceftazidime, and cefotaxime) was investigated on Mueller-Hinton agar (Oxoid, Basingstoke, UK) in the presence or absence of $10 \mathrm{mM}$ NAC. To compare the effect of NAC on antibacterial activity of $\beta$-lactam antibiotics between MRSA and MSSA, we also tested the effect of NAC on $\beta$-lactam antibacterial activity against 5 MSSA strains. The MIC of these antibiotics were determined by the E-test (Liofilchem, Roseto, Italy) method, with MIC ranging from 0.016 to $256 \mu \mathrm{g} / \mathrm{mL}$. All experiments were carried out at least in duplicate. Staphylococcus aureus ATCC29213 was used as a reference strain for the E-test. Statistical analysis was carried out in SPSS version 19.0 (IBM Corp., Armonk, NY) using T-test.

The results are summarized in Table 1 . In the presence of $10 \mathrm{~m} M \mathrm{NAC}$, the MIC of all $\beta$-lactam antibiotics to MRSA decreased remarkably. Interestingly, the MIC of oxacillin and cefoxitin in MRSA were lower than the corresponding susceptible breakpoint ( $\geq 2$ and $\geq 4 \mu \mathrm{g} /$ $\mathrm{mL}$ ) recommended by CLSI (CLSI, 2013), which means these MRSA strains were sensitive to methicillin in the presence of NAC. In addition, NAC decreased the MIC of penicillins (penicillin, oxacillin, and ampicillin) to $\leq 2.1 \%$ in MRSA, a much larger reduction than that in MSSA (5.9\% to 50.0\%). Similarly, NAC reduced the MIC of cephalosporins (cefoxitin, ceftazidime, and cefo- 


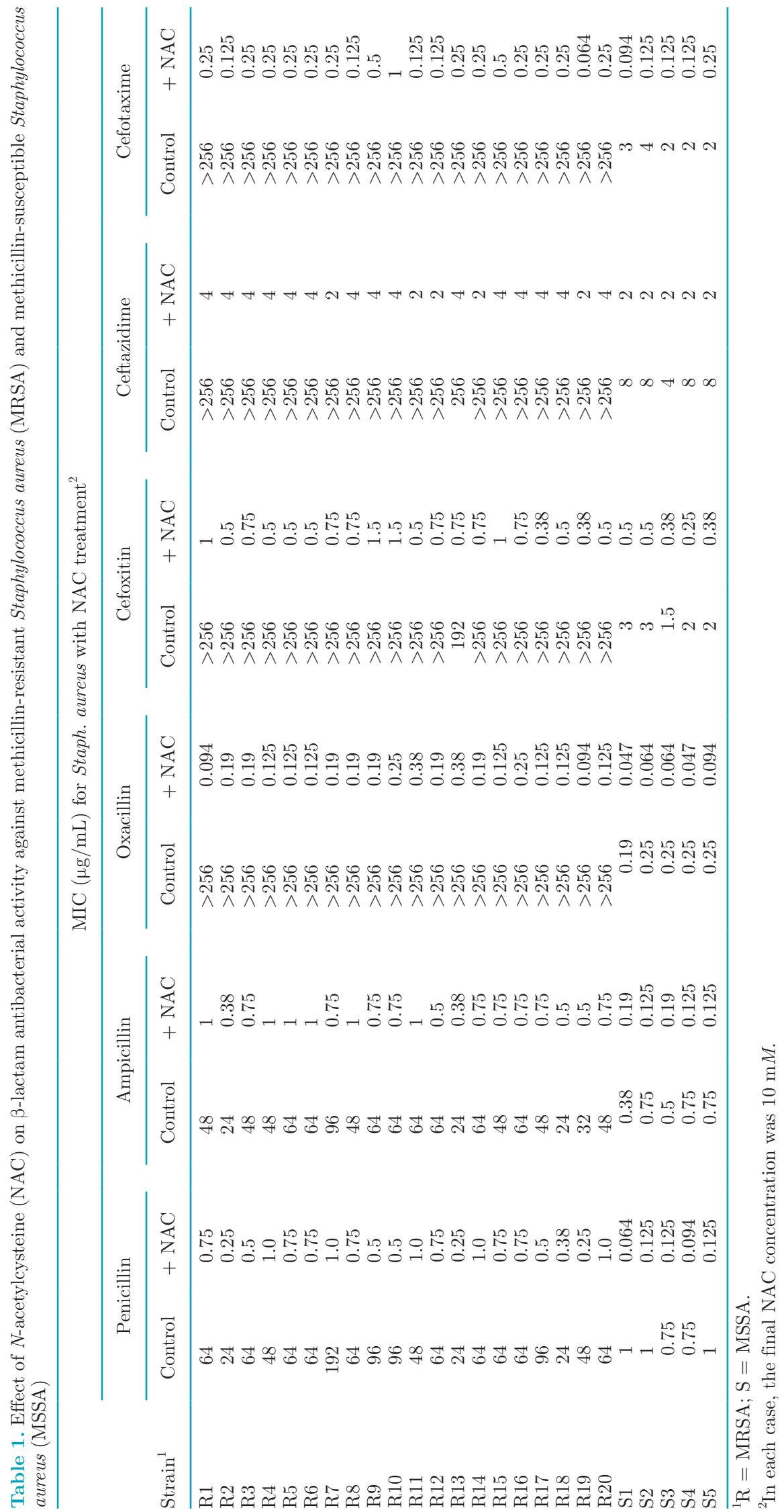


taxime) to $3.1 \%$ to $50.0 \%$ in MSSA, a smaller decrease than that in MRSA $(\leq 1.6 \%)$.

The mechanisms of antibiotic susceptibility modulation by NAC are not yet clear. Aslam et al. (2007) found that NAC made the biofilm-associated bacteria more susceptible to tigecycline by degrading the extracellular polysaccharide matrix of biofilm. Rodríguez-Beltrán et al. (2015) reported that NAC-based modulation of imipenem activity is dependent mainly on outer membrane porin D (OprD). However, these theories cannot reasonably explain the contrasting changes in our previous study, in which we reported that NAC reduced the MIC of penicillin and ampicillin but increased the MIC of erythromycin, kanamycin, and ciprofloxacin to Staph. aureus (Yang et al., 2016). In the current study, NAC markedly increased $\beta$-lactam antibacterial activity against MRSA. Meanwhile, NAC-mediated modulation of MIC in MRSA was higher than that in MSSA. It is known that MRSA and MSSA strains and isolates have different resistant mechanisms to $\beta$-lactam antibiotics (Lowy, 2003). Methicillin resistance in Staph. aureus is usually conferred by the acquisition of a non-native gene mecA encoding an altered penicillin-binding protein (PBP2a) or variant mecC encoding PBP2a $\mathrm{a}_{\mathrm{LGA}}$. Both altered proteins have extremely low affinity for most $\beta$-lactam antibiotics (Lee et al., 2018). Thus, we speculate that NAC-based modulation of $\beta$-lactam antibacterial activity to MRSA may depend on the methicillin-associated resistance gene ( and corresponding products penicillin-binding protein $\left(\mathrm{PBP} 2 \mathrm{a}\right.$ or $\left.\mathrm{PBP} 2 \mathrm{a}_{\mathrm{LGA}}\right)$. The specific mechanisms are being explored in our laboratory.

This study provides new perspectives for the use of NAC combined with $\beta$-lactam antibiotics against MRSA, but further in vivo studies are required to evaluate the effectiveness, target animal safety, and withholding periods of these combinations.

\section{ACKNOWLEDGMENTS}

This work was supported by the National Key Research and Development Program of China (grant no. 2017YFD0502200), Lanzhou; the National Natural Science Foundation of China (grant no. 31802232), Lanzhou; the Supervision of Quality and Safety of Agricultural Products (grant no. 2130109), Lanzhou; and the Science and Technology Project of Lanzhou City (grant no. 2016-3-99), Lanzhou.

\section{REFERENCES}

Aslam, S., B. W. Trautner, V. Ramanathan, and R. O. Darouiche. 2007. Combination of tigecycline and N-acetylcysteine reduces biofilm-embedded bacteria on vascular catheters. Antimicrob. Agents Chemother. 51:1556-1558.

CLSI. 2013. Performance standards for antimicrobial susceptibility testing. CLSI document M100-S23. Clinical and Laboratory Standards Institute (CLSI), Wayne, PA.

Goswami, M., and N. Jawali. 2010. N-Acetylcysteine-mediated modulation of bacterial antibiotic susceptibility. Antimicrob. Agents Chemother. 54:3529-3530.

Graveland, H., B. Duim, E. van Duijkeren, D. Heederik, and J. A. Wagenaar. 2011. Livestock-associated methicillin-resistant Staphylococcus aureus in animals and humans. Int. J. Med. Microbiol. 301:630-634.

Lee, A. S., H. D. Lencastre, J. Garau, J. Kluytmans, S. Malhotrakumar, A. Peschel, and S. Harbarth. 2018. Methicillin-resistant Staphylococcus aureus. Nat. Rev. Dis. Primers 4:18033.

Lowy, F. D. 2003. Antimicrobial resistance: The example of Staphylococcus aureus. J. Clin. Invest. 111:1265-1273.

Rodríguez-Beltrán, J., G. Cabot, E. Y. Valencia, C. Costas, G. Bou, A. Oliver, and J. Blázquez. 2015. N-Acetylcysteine selectively antagonizes the activity of imipenem in Pseudomonas aeruginosa by an OprD-mediated mechanism. Antimicrob. Agents Chemother. 59:3246-3251.

Vanderhaeghen, W., T. Cerpentier, C. Adriaensen, J. Vicca, K. Hermans, and P. Butaye. 2010. Methicillin-resistant Staphylococcus aureus (MRSA) ST398 associated with clinical and subclinical mastitis in Belgian cows. Vet. Microbiol. 144:166-171.

Yang, F., L. H. Liu, X. P. Li, J. Y. Luo, Z. Zhang, Z. T. Yan, S. D. Zhang, and H. S. Li. 2016. Short communication: N-acetylcysteinemediated modulation of antibiotic susceptibility of bovine mastitis pathogens. J. Dairy Sci. 99:4300-4302. 